\title{
Towards holography for quantum mechanics
}

\author{
Romuald A. Janik \\ Institute of Physics, Jagiellonian University, \\ ul. Eojasiewicza 11, Kraków, 30-348 Poland \\ E-mail: romuald.janik@gmail.com
}

ABSTRACT: We derive a holographic description of the simplest quantum mechanical system, a $1 \mathrm{~d}$ free particle. The dual formulation uses a couple of two-dimensional topological abelian BF theories with appropriate boundary conditions, interactions and constraints. The aim of this construction is not to use holography as a tool for quantum mechanics but rather to find the simplest possible setup in order to explore holography.

KEYWORDS: AdS-CFT Correspondence, Gauge-gravity correspondence

ARXIV EPRINT: 1805.03606 


\section{Contents}

1 Introduction 1

2 The main features of a holographic description 2

3 A holographic description of a quantum mechanical free particle $\quad 3$

$\begin{array}{lll}4 & \text { Conclusions } & 7\end{array}$

\section{Introduction}

The AdS/CFT correspondence [1] is one of the most fascinating theoretical breakthroughs in recent years. In its original form it postulated the duality between two completely different theories - the supersymmetric gauge theory $\mathcal{N}=4$ Super-Yang-Mills theory in 4 dimensions and superstring theory on a $A d S_{5} \times S^{5}$ background. Since then it has been extended in numerous directions. Apart from very important practical applications as a tool for learning about the nonperturbative dynamics of gauge theory it is particularly fascinating theoretically as it proposes the equivalence of a nongravitational theory (the gauge theory "on the boundary") and quantum theory incorporating gravity. This is a very explicit realization of the holographic principle $[2,3]$. However the very reason for which the AdS/CFT correspondence is so useful a tool for studying nonperturbative gauge theory physics makes it difficult to understand its origin microscopically from the gauge theory point of view. Indeed both sides of the duality become simple in opposite limits. In particular we do not know how to deal with string theory in the quantum gravity regime corresponding to small coupling and finite number of colors on the gauge theory side. From the point of view of understanding holography the optimal setup would be to have relatively simple and tractable quantum theories on both sides of the duality.

Some particularly intriguing generalizations of holography involved three dimensional free $O(N)$ vector model which was proposed to be dual to four dimensional Vasiliev gravity $[4,5]$. A lot of progress was made in the understanding of dual dynamics from the boundary theory point of view (see e.g. $[6,7]$ ), however the gravitational side is basically understood only at the classical and semi-classical level as Vasiliev gravity [8] has not been quantized so far.

Reducing the number of dimensions, a class of two dimensional CFT's was proposed to be dual to three dimensional Vasiliev gravity coupled to a scalar field [9]. In this case there is an explicit action for the Vasiliev theory which is a difference of two Chern-Simons theories, however the total action incorporating interactions with the scalar field is unknown and it is very difficult to study the duality in the finite $k$, finite $N$ case. $^{1}$

\footnotetext{
${ }^{1}$ In this case $k$ and $N$ are the parameters of the 2D coset CFT's.
} 
Most recently the Sachdev-Ye-Kitaev (SYK) model [10-12] (see also [13] and subsequent developments) became intensively studied as it is a quantum mechanical system which exhibits properties characteristic of a dual holographic classical gravity description in terms of black holes.

In another line of investigation, it was realized that entanglement is crucially connected with holography. Surprising parallels were uncovered between the description of ground state wave functions using MERA (Multi-scale Entanglement Renormalization Ansatz) $[14,15]$ and the Ryu-Takayanagi holographic prescription for computing entanglement entropy [16]. More recently various models for holography were proposed incorporating various tensor network constructions in particular the HaPPY proposal taking into account spatial error correcting features of the holographic dictionary [17]. Other recent advances include a path integral optimization framework [18] and the random tensor networks [19].

One generic feature of the approach to understand holography in terms of tensor networks is that these constructions are in a sense very kinematical. E.g. the HaPPY proposal provides a mapping of a boundary Hilbert space to a bulk Hilbert space which is quite agnostic about the dynamics (Hamiltonian/action etc.) of the boundary theory. If this intuition is true, it suggests that a holographic description should be in principle applicable to almost any system. ${ }^{2}$

In this short note we would like to investigate whether one can formulate a holographic dual model for the arguably simplest possible quantum system - a free particle in 1 dimension. If successful, this could be a starting point of studying more complicated setups with more degrees of freedom, interactions etc. in a context which is very much under control.

The plan of the paper is as follows. First we review some very basic requirements for a holographic description of a given theory and for identifying a gravitational subsector of the holographic bulk theory. Then we proceed to implement this program for the quantum mechanical free particle. We close the paper with a summary and conclusions.

\section{The main features of a holographic description}

In this section we will summarize what we would expect from a holographic description of some theory. Suppose that the field theory in question is defined in $d$ spacetime dimensions on some fixed nondynamical geometry $\Sigma$.

I. The dual holographic theory should be defined on a higher dimensional manifold $M$, having $\Sigma$ as a boundary. At the very least we should be able to match partition functions for the two theories

$$
Z_{\text {boundary }}=Z_{\text {bulk }}
$$

\footnotetext{
${ }^{2}$ By a holographic description we mean throughout this paper a generic higher dimensional dual description which may be very quantum and far from a description in terms of classical gravity. So we use the term in a much wider sense than e.g. in [20].
} 
II. The above requirement is not really enough as we should expect to be able to link all correlation functions in the boundary theory to the bulk theory through the GKP formula $[21,22]$. Observables/operators in the boundary theory should be associated to fields in the bulk theory. Moreover the corresponding sources in the generating function of correlators in the boundary theory should be linked to the boundary values of the associated bulk fields ${ }^{3}$ namely

$$
\int D \phi e^{i S_{\mathrm{bndry}}(\phi)+i \int_{\Sigma} j\left(x^{\mu}\right) O\left(x^{\mu}\right) d^{d} x}=Z_{\mathrm{bulk}}\left(\Phi_{O}\left(z, x^{\mu}\right) \underset{z \rightarrow 0}{\longrightarrow} j\left(x^{\mu}\right)\right) .
$$

Ultimately the boundary degrees of freedom would have been integrated out and the remaining vestiges of the boundary theory would be just the sources i.e. boundary values of the bulk fields.

III. Finally we would like to interpret a part of the bulk theory as a gravitational theory. In all holographic constructions so far, the bulk metric is the field associated to the energy momentum tensor of the boundary theory. In other words its boundary values should be linked in some way ${ }^{4}$ to the nondynamical metric of the boundary theory. Of course, as in the case of higher spin gravity the whole picture may be more complex with other massless higher spin fields making the geometric interpretation ambiguous, but still in this way we may identify a natural gravitational subsector of the bulk theory.

\section{A holographic description of a quantum mechanical free particle}

The goal of this note is to try to satisfy the above requirements for one of the simplest systems possible, the quantum mechanical free particle in one dimension. A-priori it is not at all clear if such a description exists for such a simple system. If it does exist, it may well be that the outcome is too trivial and restricted, but we hope that even such failure may be instructive and interesting as it may indicate a sharpening of the requirements for holography with respect to the ones outlined in the preceding section. From another perspective it may be a starting point for constructing holography for more nontrivial quantum mechanical systems.

This system can be understood as a QFT with no spatial dimension with the action

$$
S=\int d t \frac{1}{2} \dot{q}^{2}
$$

Since this system as it stands does not have any coupling or large $N$ parameter we expect the dual bulk theory to be necessarily quantum. This is in fact one of the key motivations of this study. We will now build up the bulk theory in steps in order to satisfy the three requirements described in section 2 .

\footnotetext{
${ }^{3}$ For simplicity we ignore potential $z^{\Delta}$ factors and assume that they have been incorporated in a redefinition of the bulk fields.

${ }^{4}$ We are purposefully quite vague about the details here. In standard AdS/CFT the dictionary is clearest in the Fefferman-Graham coordinates [23]. We do not want to impose a-priori any specific prescription in the general case.
} 
The partition function. Let us consider a two-dimensional abelian BF theory defined on the half plane

$$
M=\{(t, z): z \geq 0\}
$$

The action is given by

$$
S_{\mathrm{BF}}=\int_{M} B d A=\int B\left(\partial_{t} A_{z}-\partial_{z} A_{t}\right) d t d z
$$

We would like to impose the following boundary conditions:

$$
B=-\left.A_{t}\right|_{z=0} \quad A_{t}=\left.0\right|_{z \rightarrow \infty} .
$$

In order for these boundary conditions to be consistent with the variational principle we have to add to the action a boundary term

$$
S_{\mathrm{bulk}}^{I}=S_{\mathrm{BF}}+\frac{1}{2} \int_{\{z=0\}} B^{2} d t .
$$

The variation of the action is now

$$
\delta S_{\text {bulk }}^{I}=(\text { EOM's })+\int_{\{z=0\}} B \delta A_{t} d t+\int_{\{z=0\}} B \delta B d t
$$

which vanishes due to the boundary condition $\delta A_{t}+\delta B=\left.0\right|_{z=0}$. The superscript on $S_{\text {bulk }}^{I}$ indicates that this will not be the full final bulk action but will be still modified in the following sections.

Let us now evaluate the bulk action $S_{\text {bulk }}^{I}$. The Lagrange multiplier field $B$ imposes the constraint that $A$ is a flat connection, hence we may set

$$
A_{z}=-\partial_{z} \Phi \quad A_{t}=-\partial_{t} \Phi .
$$

The bulk part of the action $S_{\text {bulk }}^{I}$ on the constraint surfaces vanishes and we are left with just the boundary term given through the $B$ field, which in turn due to our boundary conditions can be expressed in terms of the temporal derivatives of the boundary values of $\Phi(t, z)$ field

$$
\dot{q}(t)=\lim _{z \rightarrow 0} \partial_{t} \Phi(t, z) .
$$

We thus reproduce the quantum mechanical free particle action. ${ }^{5}$

$$
\int d t \frac{1}{2} \dot{q}^{2}
$$

The above simple derivation is a two-dimensional analog of the three-dimensional link of Chern-Simons and 2d WZW [26, 27], in the variant where the boundary conditions are $A_{+}=\bar{A}_{-}=0$ (see e.g. [28]).

\footnotetext{
${ }^{5}$ Similar computations as in this subsection have been done independently with different motivations in $[24,25]$ in the case of nonabelian BF theories.
} 
Source for $q(t)$. Let us now generalize the construction by adding a generic time dependent source for the particle position $q(t)$. We thus have to reproduce an additional term in the boundary action

$$
\int d t \frac{1}{2} \dot{q}^{2}+\int d t j(t) q(t)
$$

In terms of the BF theory gauge field, the particle position $q(t)$ can be understood essentially as a Wilson line extending from the boundary to the interior at $z=\infty$ as we have

$$
\int_{z=0}^{\infty} A_{z} d z=-\int_{z=0}^{\infty} \partial_{z} \Phi(t, z)=\Phi(t, 0)-\Phi(t, \infty)
$$

Now due to the boundary condition at infinity $A_{t}=\left.0\right|_{z \rightarrow \infty}, \Phi(t, \infty)$ is a constant and hence without loss of generality can be set to zero. Therefore we can make an identification

$$
q(t)=\int_{L} A
$$

where the line $L$ is attached to the boundary at time $t$ and goes to infinity in the bulk. Now we would like to rewrite the integral

$$
\int d t j(t) q(t)
$$

as a two dimensional integral in terms of natural bulk quantities. We will also need a bulk field associated to the boundary source $j(t)$.

To this end, we will introduce another two-dimensional abelian BF theory which we will denote by

$$
\int C d \alpha
$$

In order to write the coupling (3.13) we will introduce yet another ingredient: a globally defined 1-form in the bulk which we will denote by $d t$ (for the moment this can be understood as a gradient of the $t$ coordinate). A-priori the existence of such 1-form in the context of nonrelativistic quantum mechanics is quite natural in view of Galilean symmetry. We will, however, return to this point in the following section. For the moment we will treat the 1 -form $d t$ as fixed and given externally as a gradient of the global bulk $t$ coordinate.

We will now enlarge the bulk action to

$$
S_{\mathrm{bulk}}^{I I}=\int_{M} B d A+C d \alpha+\alpha \wedge A+D \alpha \wedge d t+\frac{1}{2} \int_{\partial M} B^{2} d t
$$

Integrating over the Lagrange multiplier $D$ restricts the general form of the $\alpha$ 1-form:

$$
\alpha=j(t, z) d t
$$

Subsequently integrating over $C$ ensures that $j(t, z)$ is only a function of $t$ :

$$
\alpha=j(t) d t
$$


Now we may evaluate the bulk interaction term between the gauge fields of the two BF theories:

$$
\int_{M} \alpha \wedge A=\int_{M} j(t) d t \wedge\left(A_{t} d t+A_{z} d z\right)=\int j(t) \int_{0}^{\infty} A_{z} d z d t=\int j(t) q(t) d t
$$

obtaining exactly the boundary source term for $q(t)$.

In principle we should now perform the path integral over $A$ leaving an effective bulk action depending on the scalar fields $B, C, D$ and gauge field $\alpha$. We will not attempt to do this in this work but rather we will return to the 1-form $d t$.

Covariantizing $\boldsymbol{d} \boldsymbol{t}$ and the "gravity" subsector. Since the quantum mechanical path integral is essentially just a QFT on a 1-dimensional worldline, one can introduce a fixed 1-dimensional metric $g_{t t}(t)$ and write the action as

$$
\frac{1}{2} \int \sqrt{g} g^{t t}\left(\partial_{t} q\right)^{2}=\frac{1}{2} \int \frac{1}{e} \dot{q}^{2}
$$

where we introduced the standard einbein notation, and $e=e(t)$ is a fixed given function of time.

We would now like to complete the program sketched in section 2 and introduce a bulk field which would go over to the einbein on the boundary. At the same time we will get rid of the rather artificial looking external 1-form $d t$ which was necessary to write the boundary source term in terms of bulk fields. Since $d t$ understood as the gradient of the global bulk time coordinate is necessarily a closed 1-form, it is extremely suggestive to consider it as a gauge field of a third abelian BF theory which we will denote by

$$
\int E d \eta
$$

As the boundary condition at the physical boundary $z=0$ we will fix the temporal component of $\eta$

$$
\eta=\eta_{t} d t+\eta_{z} d z
$$

to a fixed value which we will identify shortly with the eibein $e(t)$. More precisely we fix the pullback of $\eta$ to the boundary $\partial M$ to be equal to $e(t) d t$. Thus in the case of (3.20) (as well as for (3.14)) we do not need to add any boundary terms to the action as was the case for the original $\int B d A$ theory. We will also modify the boundary conditions (3.4) at $z=0$ to

$$
A_{t}+\eta_{t} B=\left.0\right|_{z=0}
$$

Accordingly we need to modify the additional boundary term

$$
\frac{1}{2} \int_{\{z=0\}} B^{2} d t \longrightarrow \frac{1}{2} \int_{\partial M} B^{2} \eta .
$$

The cancellation of the boundary terms in the variational principle goes through since due to our boundary conditions $\delta \eta_{t}=\left.0\right|_{z=0}$. The resulting boundary action can be seen to be

$$
\frac{1}{2} \int_{\partial M} B^{2} \eta=\frac{1}{2} \int \frac{1}{\eta_{t}} A_{t}^{2} d t=\frac{1}{2} \int \frac{1}{\eta_{t}} \dot{q}^{2}
$$


where we used (3.22). It is now clear that we have to identify the boundary value of $\eta_{t}$ with the einbein $e(t)$ as announced earlier. From the considerations of section 2 we are led to identify the $E, \eta$ subsector as a part of the "gravitational" sector of the bulk theory. Note that although this is a two dimensional BF theory it is distinct from Jackiw-Teitelboim 2D gravity which is a nonabelian BF theory $[29,30]$.

Let us now put together all ingredients introduced so far. Our final bulk action takes the form

$$
S_{\mathrm{bulk}}^{I I I}=\int_{M} B d A+C d \alpha+E d \eta+\alpha \wedge A+D \alpha \wedge \eta+\frac{1}{2} \int_{\partial M} B^{2} \eta
$$

with the boundary conditions at $z=0$

$$
A_{t}+\eta_{t} B=\left.0\right|_{z=0} \quad \alpha_{t}=\left.j(t)\right|_{z=0} \quad \eta_{t}=\left.e(t)\right|_{z=0} .
$$

Let us make some comments on the above expression. Increasing the number of degrees of freedom will increase the number of components of all fields except $\eta$ and $E$. Adding interactions (on the quantum mechanical side) is rather nontrivial. One can either integrate over the source or introduce separate sources for the monomials $q(t)^{n}$. Doing that seems to require a significant extension to the formalism. Ultimately we would also like to integrate out $A$ and possibly $B$. We leave these issues for future investigation.

\section{Conclusions}

The motivation for the construction presented in this note is the intuition arising from tensor network interpretations of holography that a holographic description should exist for almost any system. Hence it should be possible to find a holographic formulation of the most extreme simple system that one could think of - a one dimensional quantum mechanical free particle. As we would like to have an explicit dual theory described by some concrete bulk action, we did not take the approach through tensor network constructions but rather we worked directly in the continuum with two dimensional topological BF theories having the Chern-Simons/WZW relation as a guiding principle. The expected features of a holographic dual imposes, however, further requirements on the bulk theory going beyond the equality of partition functions. In particular we should have additional matter fields in the bulk theory which are associated to the operators of the boundary theory and which reduce to the corresponding sources at the boundary. In this work we carried out the construction for the source for the particle position $q(t)$. We also identified a subsector of the bulk theory which reduces to the einbein on the boundary and thus behaves like a "gravitational" sector of the bulk theory.

A characteristic feature of the simple quantum mechanical model considered here is the absence of a large $N$ parameter. More precisely, one can consider this model to have $N=1$, with a straightforward generalization to $N$ components. In the conventional examples of the AdS/CFT correspondence, finite $N$ corresponds to a quantum bulk model (in these cases quantum gravity+other matter fields), which was also a motivation for the present construction, where we treat the bulk theory on the quantum level as we use the full 
path integral formalism. Indeed the role of a large $N$ limit in a generalized version of the model (possibly with a singlet constraint) within a similar construction is a very interesting problem which we plan to address in the future.

One qualitative feature of holography which is not explicitly captured by the present construction is the interpretation of the holographic direction as an RG flow. In the present paper, on the other hand, the starting point of the construction was a minimal implementation of the bulk formula for the generating function of correlators (2.2), which does not lead to a direct RG interpretation (which in any case is not evident as the quantum mechanical system lives on a worldline and thus has no spatial dimension). We suspect that to address this issue one would have to integrate out the $A$ and $B$ fields and analyze the resulting theory of just the bulk fields associated with sources of $q(t)$ and the einbein $e(t)$. Possibly for a local geometric interpretation one would have to combine this procedure with the large $N$ limit discussed above. This goes beyond the scope of the present paper but is definitely another important problem for future research.

There are also many other possible directions for further investigation, foremost of which is going to nontrivial quantum mechanical systems. It is not completely clear whether to consider in addition sources for monomials of $q(t)$ and to what extent the construction of the source sector performed here is unique or optimal. On a more mundane level it would be interesting to analyze the bulk theory in more detail and check to what extent our experience with holography in higher number of dimensions carries through here. We hope that the setup presented in this paper would be a good framework to address such questions.

\section{Acknowledgments}

This work was supported by NCN grant 2012/06/A/ST2/00396.

Open Access. This article is distributed under the terms of the Creative Commons Attribution License (CC-BY 4.0), which permits any use, distribution and reproduction in any medium, provided the original author(s) and source are credited.

\section{References}

[1] J.M. Maldacena, The Large $N$ limit of superconformal field theories and supergravity, Int. J. Theor. Phys. 38 (1999) 1113 [Adv. Theor. Math. Phys. 2 (1998) 231] [hep-th/9711200] [INSPIRE].

[2] G. 't Hooft, Dimensional reduction in quantum gravity, Conf. Proc. C 930308 (1993) 284 [gr-qc/9310026] [INSPIRE].

[3] L. Susskind, The World as a hologram, J. Math. Phys. 36 (1995) 6377 [hep-th/9409089] [INSPIRE].

[4] I.R. Klebanov and A.M. Polyakov, AdS dual of the critical $O(N)$ vector model, Phys. Lett. B 550 (2002) 213 [hep-th/0210114] [INSPIRE].

[5] S. Giombi and X. Yin, Higher Spin Gauge Theory and Holography: The Three-Point Functions, JHEP 09 (2010) 115 [arXiv:0912.3462] [INSPIRE]. 
[6] R. de Mello Koch, A. Jevicki, K. Jin and J.P. Rodrigues, $A d S_{4} / C F T_{3}$ Construction from Collective Fields, Phys. Rev. D 83 (2011) 025006 [arXiv: 1008.0633] [InSPIRE].

[7] J.R. Fliss, R.G. Leigh and O. Parrikar, Unitary Networks from the Exact Renormalization of Wave Functionals, Phys. Rev. D 95 (2017) 126001 [arXiv: 1609.03493] [INSPIRE].

[8] M.A. Vasiliev, Higher spin gauge theories in four-dimensions, three-dimensions and two-dimensions, Int. J. Mod. Phys. D 5 (1996) 763 [hep-th/9611024] [INSPIRE].

[9] M.R. Gaberdiel and R. Gopakumar, An AdS 3 Dual for Minimal Model CFTs, Phys. Rev. D 83 (2011) 066007 [arXiv: 1011.2986] [INSPIRE].

[10] S. Sachdev and J. Ye, Gapless spin fluid ground state in a random, quantum Heisenberg magnet, Phys. Rev. Lett. 70 (1993) 3339 [cond-mat/9212030] [INSPIRE].

[11] A. Kitaev, A simple model of quantum holography. Part 1, talk given at KITP, University of California, Santa Barbara, California, U.S.A., 7 April 2015 and online at http://online.kitp.ucsb.edu/online/entangled15/kitaev/.

[12] A. Kitaev, A simple model of quantum holography. Part 2, talk given at KITP, University of California, Santa Barbara, California, U.S.A., 27 May 2015 and online at http://online.kitp.ucsb.edu/online/entangled15/kitaev2/.

[13] J.M. Maldacena and D. Stanford, Remarks on the Sachdev-Ye-Kitaev model, Phys. Rev. D 94 (2016) 106002 [arXiv: 1604.07818] [INSPIRE].

[14] B. Swingle, Entanglement Renormalization and Holography, Phys. Rev. D 86 (2012) 065007 [arXiv: 0905.1317] [INSPIRE].

[15] M. Nozaki, S. Ryu and T. Takayanagi, Holographic Geometry of Entanglement Renormalization in Quantum Field Theories, JHEP 10 (2012) 193 [arXiv:1208.3469] [INSPIRE].

[16] S. Ryu and T. Takayanagi, Holographic derivation of entanglement entropy from AdS/CFT, Phys. Rev. Lett. 96 (2006) 181602 [hep-th/0603001] [INSPIRE].

[17] F. Pastawski, B. Yoshida, D. Harlow and J. Preskill, Holographic quantum error-correcting codes: Toy models for the bulk/boundary correspondence, JHEP 06 (2015) 149 [arXiv: 1503.06237] [INSPIRE].

[18] P. Caputa, N. Kundu, M. Miyaji, T. Takayanagi and K. Watanabe, Anti-de Sitter Space from Optimization of Path Integrals in Conformal Field Theories,

Phys. Rev. Lett. 119 (2017) 071602 [arXiv:1703.00456] [INSPIRE].

[19] P. Hayden, S. Nezami, X.-L. Qi, N. Thomas, M. Walter and Z. Yang, Holographic duality from random tensor networks, JHEP 11 (2016) 009 [arXiv: 1601.01694] [INSPIRE].

[20] I. Heemskerk, J. Penedones, J. Polchinski and J. Sully, Holography from Conformal Field Theory, JHEP 10 (2009) 079 [arXiv:0907.0151] [INSPIRE].

[21] S.S. Gubser, I.R. Klebanov and A.M. Polyakov, Gauge theory correlators from noncritical string theory, Phys. Lett. B 428 (1998) 105 [hep-th/9802109] [INSPIRE].

[22] E. Witten, Anti-de Sitter space and holography, Adv. Theor. Math. Phys. 2 (1998) 253 [hep-th/9802150] [INSPIRE].

[23] S. de Haro, S.N. Solodukhin and K. Skenderis, Holographic reconstruction of space-time and renormalization in the AdS/CFT correspondence, Commun. Math. Phys. 217 (2001) 595 [hep-th/0002230] [INSPIRE]. 
[24] T.G. Mertens, The Schwarzian Theory - Origins, JHEP 05 (2018) 036 [arXiv: 1801.09605] [INSPIRE].

[25] H.A. González, D. Grumiller and J. Salzer, Towards a bulk description of higher spin SYK, JHEP 05 (2018) 083 [arXiv: 1802.01562] [INSPIRE].

[26] E. Witten, Quantum Field Theory and the Jones Polynomial, Commun. Math. Phys. 121 (1989) 351 [INSPIRE].

[27] S. Elitzur, G.W. Moore, A. Schwimmer and N. Seiberg, Remarks on the Canonical Quantization of the Chern-Simons-Witten Theory, Nucl. Phys. B 326 (1989) 108 [InSPIRE].

[28] O. Coussaert, M. Henneaux and P. van Driel, The Asymptotic dynamics of three-dimensional Einstein gravity with a negative cosmological constant, Class. Quant. Grav. 12 (1995) 2961 [gr-qc/9506019] [INSPIRE].

[29] D. Cangemi and R. Jackiw, Gauge invariant formulations of lineal gravity, Phys. Rev. Lett. 69 (1992) 233 [hep-th/9203056] [inSPIRE].

[30] R. Jackiw, Two lectures on two-dimensional gravity, in proceedings of the 2nd Latin American School on Theoretical Physics: Strings and Fundamentals (LASSF II), Caracas, Venezuela, 23-27 October 1995, gr-qc/9511048 [INSPIRE]. 